\title{
Doubly Constrained Robust Blind Beamforming Algorithm
}

\author{
Xin Song, Jingguo Ren, and Qiuming Li \\ Engineering Optimization and Smart Antenna Institute, Northeastern University at Qinhuangdao, Qinhuangdao 066004, China
}

Correspondence should be addressed to Xin Song; songxin00@126.com

Received 28 December 2012; Accepted 15 July 2013

Academic Editor: Bin Wang

Copyright (C) 2013 Xin Song et al. This is an open access article distributed under the Creative Commons Attribution License, which permits unrestricted use, distribution, and reproduction in any medium, provided the original work is properly cited.

\begin{abstract}
We propose doubly constrained robust least-squares constant modulus algorithm (LSCMA) to solve the problem of signal steering vector mismatches via the Bayesian method and worst-case performance optimization, which is based on the mismatches between the actual and presumed steering vectors. The weight vector is iteratively updated with penalty for the worst-case signal steering vector by the partial Taylor-series expansion and Lagrange multiplier method, in which the Lagrange multipliers can be optimally derived and incorporated at each step. A theoretical analysis for our proposed algorithm in terms of complexity cost, convergence performance, and SINR performance is presented in this paper. In contrast to the linearly constrained LSCMA, the proposed algorithm provides better robustness against the signal steering vector mismatches, yields higher signal captive performance, improves greater array output SINR, and has a lower computational cost. The simulation results confirm the superiority of the proposed algorithm on beampattern control and output SINR enhancement.
\end{abstract}

\section{Introduction}

Adaptive beamforming, as an attractive solution to signal detection and estimation in harsh environments, has received considerable attention in the fields of radar, sonar, seismology, radio astronomy, medical imaging, artificial intelligence, and neural network [1-5]. Many methods have been proposed and received great attention in the last twenty years. The class of blind adaptive beamforming algorithms is generally known as LSCMA, which can be rapidly convergent and globally stable for any linearly independent set of input signals. However, in the complex communication environments, adaptive beamforming algorithms may suffer significant performance degradation in the presence of the signal mismatches between the actual and assumed signal steering vectors. Such a type of mismatches may occur due to the unknown deformation of the antenna or sensor array, steer direction errors, and the drifting effect in the electronics or the multipath propagation, and so forth. Many solutions have been proposed such as convex quadratic constraints [6] and Bayesian approach [7] to account for the steering direction error of the target source. The eigenspace-based algorithm $[8,9]$ is also a good approach for robust beamforming. However, it is inefficient at low input signal-to-noise ratio (SNR) due to the substantial possibility of subspace swap. Recently, some advanced methods have been proposed [10-19]. It is proven that many robust adaptive beamformers belong to the family of the diagonal loading method. With the generalization of the sphere uncertainty set to ellipsoid, the optimal diagonal loading level can be calculated efficiently by the proposed methods $[10,11]$. A very effective approach developed to the design of robust adaptive beamforming is based on the principle of worst-case performance optimization [12-14]. This approach delimits the uncertainty set of steering vectors by upper-bounding the norm of the difference between the actual and presumed steering vectors, that is, the norm of the mismatch vector. The value of the upper bound is assumed to be known. Regrettably, the second-order cone programming (SOCP) method does not provide a closed-form solution for the weight vector, and even it cannot be implemented online [15]. The general rank case has been considered, and an elegant closed-form solution has been obtained [16]. In a multiuser multipleinput single-output (MISO) cognitive radio network, the design of robust downlink beamforming is presented [17]. To reduce the impact of the channel state information (CSI) errors, two robust beamforming schemes are proposed in [18], which recover the large fraction of the SINR lost due to the channel estimation errors, but ultimately a large enough 
channel mismatch can eliminate the secrecy advantage of using artificial noise. To mitigate the detrimental effect of interferers, we extend the one-dimensional covariance fitting approach to multidimensional covariance fitting, modeling the source steering vector by means of uncertainty sets [19].

In this paper, robust LSCMA based on double constraints is proposed via the worst-case performance optimization. The quadratic constraint on the weight vector can improve robustness to the signal steering vector mismatches. In order for LSCMA to provide improved performance, the updating weight vector subject to the constraints of distortionless array response is derived by the partial Taylor-series expansion and Lagrange multiplier method, in which the multipliers can be optimally derived and incorporated at each step. The implementation of the proposed algorithm based on iterative minimization eliminates the covariance matrix inversion estimation, so it has a low computational load. Compared with the linearly constrained LSCMA, the proposed algorithm suffers the least distortion from the direction near the desired steering angle, yields better signal captive performance, and has superior performance on SINR improvement. The theoretical analysis and simulation results have been carried out to demonstrate effectiveness and superiority of the proposed algorithm in the signal steering vector mismatches. So the proposed algorithm can be an appealing technique and be implemented in digital system to improve the receiver performance.

\section{Problem Formulation}

2.1. Signal Model. We assume that there are $M$ sensors and $D$ unknown sources impinging from directions $\left\{\theta_{0}, \theta_{1}, \ldots, \theta_{D-1}\right\}$. The sensors receive the linear combination of the source signals in the presence of additive white Gaussian noise (AWGN). Therefore, the received signal vector is given by

$$
\begin{aligned}
\mathbf{x}(k) & =s_{0}(k) \mathbf{a}\left(\theta_{0}\right)+\mathbf{i}(k)+\mathbf{n}(k) \\
& =\mathbf{A}_{D} \mathbf{S}(k)+\mathbf{n}(k),
\end{aligned}
$$

where $\mathbf{x}(k)=\left[x_{1}(k), x_{2}(k), \ldots, x_{M}(k)\right]^{\mathrm{T}}$ is the observed signal vector, $\mathbf{a}\left(\theta_{0}\right)$ is the desired signal steering vector, $\mathbf{A}_{D}=\left[\mathbf{a}\left(\theta_{0}\right), \mathbf{a}\left(\theta_{1}\right), \ldots, \mathbf{a}\left(\theta_{D-1}\right)\right]$ is the array manifold, $\mathbf{S}(k)$ is the vector of $D$ transmitted signals, $\mathbf{i}(k)$ is the interference components, and $\mathbf{n}(k)$ is the noise components with zero mean. The aim of blind adaptive beamforming is to estimate the source signal $s_{0}(k)$ using only the observed data $\mathbf{x}(k)$. We write the estimated source signal as

$$
y(k)=\mathbf{w}^{\mathrm{H}} \mathbf{x}(k),
$$

where $\mathbf{w}=\left[w_{1}, w_{2}, \ldots, w_{M}\right]^{\mathrm{T}}$ is the complex weight vector and $(\cdot)^{\mathrm{T}}$ and $(\cdot)^{\mathrm{H}}$ stand for the transpose and Hermitian transpose, respectively. The signal-to-interference-plus-noise ratio (SINR) has the following form:

$$
\operatorname{SINR}=\frac{\sigma_{s}^{2}\left|\mathbf{w}^{\mathrm{H}} \mathbf{a}\left(\theta_{0}\right)\right|^{2}}{\mathbf{w}^{\mathrm{H}} \mathbf{R}_{i+n} \mathbf{w}},
$$

where $\sigma_{s}^{2}$ is the signal power and $\mathbf{R}_{i+n}$ is the $M \times M$ interference-plus-noise covariance matrix:

$$
\mathbf{R}_{i+n}=E\left\{(\mathbf{i}(k)+\mathbf{n}(k))(\mathbf{i}(k)+\mathbf{n}(k))^{\mathrm{H}}\right\},
$$

where $E[\cdot]$ denotes statistical expectation.

In the array signal processing, the objective of adaptive beamforming is to enhance the desired signal and suppress the noise and interference signals, which improves the array output SINR. In the adaptive array antenna system, the output SINR achieved the optimal one by regulating the weight vector.

2.2. The Linearly Constrained LSCMA. The linearly constrained LSCMA that is an effective solution to the problem of interference capture can be used for equalization, blind adaptive beamforming, and other similar applications when the desired signal has a constant envelope [20]. It is formulated as the following optimization problem:

$$
\min \quad g(\mathbf{w})=\sum_{i}^{K}\left|f_{i}(\mathbf{w})\right|^{2}=\|\mathbf{f}(\mathbf{w})\|_{2}^{2}
$$$$
\text { subject to } \mathbf{w}^{\mathrm{H}} \mathbf{a}\left(\theta_{0}\right)=1 \text {, }
$$

where $\mathbf{f}(\mathbf{w})=\left[f_{1}(\mathbf{w}), \ldots, f_{K}(\mathbf{w})\right]^{\mathrm{T}}$ and $f_{i}(\mathbf{w})=\left|\mathbf{w}^{\mathrm{H}} \mathbf{x}(i)\right|-1$.

We define $\mathbf{X}_{K}=[\mathbf{x}(1), \mathbf{x}(2), \ldots, \mathbf{x}(K)]$ and solve (5) to obtain the weight vector

$$
\mathbf{w}(k+1)=\mathbf{w}(k)-\widehat{\mathbf{R}}_{K}^{-1}\left[\mathbf{X}_{K} \mathbf{e}^{\mathrm{H}}+\mathbf{a}\left(\theta_{0}\right) \delta\right],
$$

where

$$
\begin{gathered}
\widehat{\mathbf{R}}_{K}=\sum_{i=1}^{K} \mathbf{x}(i) \mathbf{x}^{\mathrm{H}}(i), \\
\delta=-\frac{1-\mathbf{a}^{\mathrm{H}}\left(\theta_{0}\right) \mathbf{w}(k)+\mathbf{a}^{\mathrm{H}}\left(\theta_{0}\right) \widehat{\mathbf{R}}_{K}^{-1} \mathbf{X}_{K} \mathbf{e}^{\mathrm{H}}}{\mathbf{a}^{\mathrm{H}}\left(\theta_{0}\right) \widehat{\mathbf{R}}_{K}^{-1} \mathbf{a}\left(\theta_{0}\right)}, \\
\mathbf{e}=\left[e_{1}, \ldots, e_{K}\right]=\left[y_{1}-\frac{y_{1}}{\left|y_{1}\right|}, \ldots, y_{K}-\frac{y_{K}}{\left|y_{K}\right|}\right] .
\end{gathered}
$$

From (6), we note that the constrained LSCMA requires the precise steering vector of the desired signal. But in practical applications, this may bring the mismatch between the presumed and actual signal steering vectors, because some of the underlying assumptions on the environments, sources, or sensor array can be violated. Therefore, the linearly constrained LSCMA is very sensitive to the signal steering vector mismatches, which causes serious cancellation problem of the desired signal.

\section{Robust Constrained LSCMA under Double Constraints}

To overcome the above-mentioned problem, robust constrained LSCMA is proposed, which provides excellent robustness against signal steering vector mismatches, suppresses the interference signals effectively, and enhances 
the array output SINR. In practical applications, the array beampattern error is formulated as

$$
\begin{aligned}
\vartheta^{2} & =\mathbf{w}^{\mathrm{H}}\left[\int_{\theta_{0}-\Delta \theta}^{\theta_{0}+\Delta \theta} \cos \theta(\widetilde{\mathbf{a}}-\overline{\mathbf{a}})(\widetilde{\mathbf{a}}-\overline{\mathbf{a}})^{\mathrm{H}} \mathrm{d} \theta\right] \mathbf{w} \\
& =\mathbf{w}^{\mathrm{H}} \mathbf{Q} \mathbf{w},
\end{aligned}
$$

where $\mathbf{Q}=\int_{\theta_{0}-\Delta \theta}^{\theta_{0}+\Delta \theta}\left(\widetilde{\mathbf{a}} \widetilde{\mathbf{a}}^{\mathrm{H}}-\widetilde{\mathbf{a}} \overline{\mathbf{a}}^{\mathrm{H}}-\overline{\mathbf{a}} \widetilde{\mathbf{a}}^{\mathrm{H}}+\overline{\mathbf{a}} \cdot \overline{\mathbf{a}}^{\mathrm{H}}\right) \mathrm{d} \sin \theta, \overline{\mathbf{a}}$ is the assumed steering vector, and $\widetilde{\mathbf{a}}$ is the estimated steering vector with mismatches.

The cost function of robust constrained LSCMA can be written as

$$
\min _{\mathbf{w}} g(\mathbf{w})=\sum_{i=1}^{K}|| \mathbf{w}^{\mathrm{H}} \mathbf{x}(i)|-1|^{2} \quad \text { subject to } \mathbf{w}^{\mathrm{H}} \mathbf{Q} \mathbf{w} \leq \xi^{2},
$$

where $\xi$ is a given integer. The new formulation (9) is based on the worst-case performance optimization because it implies that $g(\mathbf{w})$ is minimized subject to the constraint and the distortionless array response will be maintained for the worst-case mismatch $\Delta \theta$.

The quadratic constraint is adjoined to the cost function by the Lagrange multiplier $\eta$, and we can obtain the Lagrange function $H(\mathbf{w}, \eta)$ :

$$
H(\mathbf{w}, \eta)=\frac{1}{2} \sum_{i=1}^{K}|| \mathbf{w}^{\mathrm{H}} \mathbf{x}(i)|-1|^{2}+\frac{1}{2} \eta\left(\mathbf{w}^{\mathrm{H}} \mathbf{Q} \mathbf{w}-\xi^{2}\right) .
$$

The partial Taylor-series expansion of (10) is

$$
\begin{aligned}
H(\mathbf{w}+\mathbf{d}, \eta)= & \frac{1}{2}\left\|\mathbf{f}(\mathbf{w})+\mathbf{J}^{\mathrm{H}}(\mathbf{w}) \mathbf{d}\right\|_{2}^{2} \\
& +\frac{1}{2} \eta\left(\mathbf{w}^{\mathrm{H}} \mathbf{Q} \mathbf{w}-\xi^{2}\right)+\eta \mathbf{w}^{\mathrm{H}} \mathbf{Q}^{\mathrm{H}} \mathbf{d},
\end{aligned}
$$

where $\mathbf{d}$ is the offset vector and $\mathbf{J}(\mathbf{w})$ is the Jacobian of $\mathbf{f}(\mathbf{w})$ :

$$
\begin{gathered}
\mathbf{J}(\mathbf{w})=\nabla_{\mathbf{w}} \mathbf{f}(\mathbf{w})=\left[\nabla_{\mathbf{w}} f_{1}(\mathbf{w}), \ldots, \nabla_{\mathbf{w}} f_{K}(\mathbf{w})\right], \\
\nabla_{\mathbf{w}} f_{i}(\mathbf{w})=\nabla_{\mathbf{w}}\left\{\left|\mathbf{w}^{\mathrm{H}} \mathbf{x}(i)\right|-1\right\}=\mathbf{x}(i) \frac{y_{i}^{*}}{\left|y_{i}\right|} .
\end{gathered}
$$

Take the gradient of $H(\mathbf{w}+\mathbf{d}, \eta)$ with respect to $\mathbf{d}$ :

$$
\nabla_{\mathbf{d}}(H(\mathbf{w}+\mathbf{d}, \eta))=\mathbf{J}(\mathbf{w}) \mathbf{J}^{\mathrm{H}}(\mathbf{w}) \mathbf{d}+\mathbf{J}(\mathbf{w}) \mathbf{f}(\mathbf{w})+\eta \mathbf{Q w} .
$$

By equating (13) to zero, the offset vector $\mathbf{d}$ can be calculated as

$$
\mathbf{d}=-\left[\mathbf{J}(\mathbf{w}) \mathbf{J}^{\mathrm{H}}(\mathbf{w})\right]^{-1}[\mathbf{J}(\mathbf{w}) \mathbf{f}(\mathbf{w})+\eta \mathbf{Q w}] .
$$

Using (12), we can derive the following equation simply:

$$
\begin{gathered}
\mathbf{J}(\mathbf{w}) \mathbf{J}^{\mathrm{H}}(\mathbf{w})=\mathbf{X}_{K} \mathbf{X}_{K}^{\mathrm{H}}=\widehat{\mathbf{R}}_{K}, \\
\mathbf{J}(\mathbf{w}) \mathbf{f}(\mathbf{w})=\mathbf{X}_{K} \mathbf{e}^{\mathrm{H}} .
\end{gathered}
$$

Combining (15) and (14), we can rewrite the offset vector

$$
\mathbf{d}=-\widehat{\mathbf{R}}_{K}^{-1}\left[\mathbf{X}_{K} \mathbf{e}^{\mathrm{H}}+\eta \mathbf{Q w}\right] .
$$

Then, using (16), the updating weight vector for robust constrained LSCMA becomes

$$
\begin{aligned}
\mathbf{w}(k+1) & =\mathbf{w}(k)-\widehat{\mathbf{R}}_{K}^{-1}\left[\mathbf{X}_{K} \mathbf{e}^{\mathrm{H}}+\eta \mathbf{Q w}(k)\right] \\
& =\left(\mathbf{I}-\eta \widehat{\mathbf{R}}_{K}^{-1} \mathbf{Q}\right) \mathbf{w}(k)-\widehat{\mathbf{R}}_{K}^{-1} \mathbf{X}_{K} \mathbf{e}^{\mathrm{H}}
\end{aligned}
$$

From (6) and (17), we remark that the major computational demand to derive the weight vector comes from the covariance matrix inversion, which requires $O\left(M^{3}\right)$ flops. This leads to a high computational cost. In our proposed algorithm, to solve this problem, the iterative method is used to calculate the covariance matrix inversion. Using the matrix inverse lemma, we can obtain

$$
\begin{aligned}
\mathbf{G}(K) & =\mathbf{R}_{K}^{-1} \\
& =\left[\mathbf{G}(K-1)-\frac{\mathbf{G}(K-1) \mathbf{x}(K) \mathbf{x}^{\mathrm{H}}(K) \mathbf{G}(K-1)}{1+\mathbf{x}^{\mathrm{H}}(K) \mathbf{G}(K-1) \mathbf{x}(K)}\right] .
\end{aligned}
$$

Inserting (18) into (17), the weight vector is updated as

$$
\mathbf{w}(k+1)=(\mathbf{I}-\eta \mathbf{G}(k) \mathbf{Q}) \mathbf{w}(k)-\mathbf{G}(k) \mathbf{X}_{K} \mathbf{e}^{\mathrm{H}} .
$$

Equation (19) is substituted into the quadratic constraint in (9), which yields

$$
(\mathbf{F}(k)-\eta \mathbf{D}(k))^{\mathrm{H}} \mathbf{Q}(\mathbf{F}(k)-\eta \mathbf{D}(k))=\xi^{2},
$$

where

$$
\begin{gathered}
\mathbf{F}(k)=\mathbf{w}(k)-\mathbf{G}(k) \mathbf{X}_{K} \mathbf{e}^{\mathrm{H}}, \\
\mathbf{D}(k)=\mathbf{G}(k) \mathbf{Q} \mathbf{w}(k) .
\end{gathered}
$$

To solve (20), the Lagrange multiplier $\eta$ has the following form:

$$
\eta=\frac{\operatorname{Re}\left[\mathbf{F}^{\mathrm{H}}(k) \mathbf{Q D}(k)\right]-\operatorname{Re}[\rho(k)]}{\mathbf{D}^{\mathrm{H}}(k) \mathbf{Q D}(k)},
$$

where

$$
\begin{aligned}
\rho^{*}(k) \rho(k)= & \left(\operatorname{Re}\left[\mathbf{F}^{\mathrm{H}}(k) \mathbf{Q D}(k)\right]\right)^{2} \\
& -\left[\mathbf{D}^{\mathrm{H}}(k) \mathbf{Q D}(k)\left(\mathbf{F}^{\mathrm{H}}(k) \mathbf{Q F}(k)-\xi^{2}\right)\right] .
\end{aligned}
$$

In order to detect the desired signal under directional uncertainty, we can impose another constraint on an average steering vector via the Bayesian approach. We assume that the direction of arrival (DOA) is a discrete random variable with known a priori probability density function (pdf) $q(\widetilde{\theta})$ that reflects the level of uncertainty about the source DOA. For computational simplicity, we assume that $q(\tilde{\theta})$ is defined only 
on a discrete set of $L$ points, $\Theta=\left\{\widetilde{\theta}_{1}, \widetilde{\theta}_{2}, \ldots, \widetilde{\theta}_{L}\right\}$, in the a priori parameter space.

When interferers are present, the a posteriori probability density function $p\left(\widetilde{\theta}_{i} \mid \mathbf{X}_{K}\right)$ is difficult to implement because it is a function of $\mathbf{R}_{i+n}$, which is unknown and hard to estimate. We derive approximate $p\left(\widetilde{\theta}_{i} \mid \mathbf{X}_{K}\right)$ with a simpler expression [7]

$$
\widehat{p}\left(\widetilde{\theta}_{i} \mid \mathbf{X}_{K}\right)=\frac{q\left(\widetilde{\theta}_{i}\right) \exp \left\{\beta K\left(\mathbf{a}^{\mathrm{H}}\left(\widetilde{\theta}_{i}\right) \widehat{\mathbf{R}}_{K}^{-1} \mathbf{a}\left(\widetilde{\theta}_{i}\right)\right)^{-1}\right\}}{\sum_{j=1}^{L} q\left(\widetilde{\theta}_{j}\right) \exp \left\{\beta K\left(\mathbf{a}^{\mathrm{H}}\left(\widetilde{\theta}_{j}\right) \widehat{\mathbf{R}}_{K}^{-1} \mathbf{a}\left(\widetilde{\theta}_{j}\right)\right)^{-1}\right\}},
$$

where $\beta$ is a monotonically increasing function of SNR.

At low SNR, it will be relatively flat over all DOAs and revert to the a priori pdf. At high SNR, the a posteriori probability of the true DOA will approach one, whereas the posteriori probability of the other DOAs will approach zero.

Based on (24), we can consider that a further form of robust constrained LSCMA (9) is expressed as

$$
\min _{\mathbf{w}} \sum_{i=1}^{K}|| \mathbf{w}^{\mathrm{H}} \mathbf{x}(i)|-1|^{2} \quad \text { subject to } \mathbf{w}^{\mathrm{H}} \widehat{\mathbf{a}}_{\mathbf{v}}=1, \mathbf{w}^{\mathrm{H}} \mathbf{Q} \mathbf{w} \leq \xi^{2},
$$

where $\widehat{\mathbf{a}}_{\mathrm{v}}$ is an average steering vector averaged over $\widehat{p}\left(\widetilde{\theta}_{i}\right.$ | $\left.\mathbf{X}_{K}\right)$ :

$$
\widehat{\mathbf{a}}_{\mathbf{v}}=\sum_{i=1}^{L} \mathbf{a}\left(\widetilde{\theta}_{i}\right) \widehat{p}\left(\widetilde{\theta}_{i} \mid \mathbf{X}_{K}\right)=\mathbf{A p},
$$

where $\mathbf{A}=\left[\mathbf{a}\left(\widetilde{\theta}_{1}\right), \mathbf{a}\left(\widetilde{\theta}_{2}\right), \ldots, \mathbf{a}\left(\tilde{\theta}_{L}\right)\right]$ is the $M \times L$ matrix of steering vectors and $\mathbf{p}$ is the $L \times 1$ vector.

Gauss's method updates $\mathbf{w}$ by the offset $\hat{\mathbf{d}}$ that minimizes the partial Taylor-series expansion of (25) subject to the double constraints; that is,

$$
\begin{aligned}
& \min _{\mathbf{w}} g(\mathbf{w}+\hat{\mathbf{d}}) \approx\left\|\mathbf{f}(\mathbf{w})+\mathbf{J}^{\mathrm{H}}(\mathbf{w}) \hat{\mathbf{d}}\right\|_{2}^{2} \\
& \text { subject to } \quad \mathbf{w}^{\mathrm{H}} \widehat{\mathbf{a}}_{\mathrm{v}}=1, \mathbf{w}^{\mathrm{H}} \mathbf{Q} \mathbf{w} \leq \xi^{2} .
\end{aligned}
$$

Using the Lagrange multiplier method, the optimal solution to (27) is obtained by minimizing the following function:

$$
\begin{aligned}
\widehat{H}(\mathbf{w}+\widehat{\mathbf{d}}, \lambda, \gamma)= & \frac{1}{2}\left\|\mathbf{f}(\mathbf{w})+\mathbf{J}^{\mathrm{H}}(\mathbf{w}) \hat{\mathbf{d}}\right\|_{2}^{2}+\lambda\left(\mathbf{w}^{\mathrm{H}} \widehat{\mathbf{a}}_{\mathbf{v}}-1\right) \\
& +\frac{1}{2} \gamma\left(\mathbf{w}^{\mathrm{H}} \mathbf{Q} \mathbf{w}-\xi^{2}\right)+\lambda \widehat{\mathbf{a}}_{\mathrm{v}}^{\mathrm{H}} \widehat{\mathbf{d}}+\gamma \mathbf{w}^{\mathrm{H}} \mathbf{Q}^{\mathrm{H}} \widehat{\mathbf{d}},
\end{aligned}
$$

where $\lambda$ and $\gamma$ are the Lagrange multipliers.
The gradient of $H(\mathbf{w}+\hat{\mathbf{d}}, \lambda, \gamma)$ with respect to $\widehat{\mathbf{d}}$ is

$$
\begin{aligned}
\nabla_{\widehat{\mathbf{d}}}( & \hat{H}(\mathbf{w}+\widehat{\mathbf{d}}, \lambda, \gamma)) \\
= & \frac{1}{2} \nabla_{\widehat{\mathbf{d}}}\left\{\left[\mathbf{f}(\mathbf{w})+\mathbf{J}^{\mathrm{H}}(\mathbf{w}) \hat{\mathbf{d}}\right]^{\mathrm{H}}\left[\mathbf{f}(\mathbf{w})+\mathbf{J}^{\mathrm{H}}(\mathbf{w}) \hat{\mathbf{d}}\right]\right\} \\
& +\lambda \widehat{\mathbf{a}}_{\mathbf{v}}+\gamma \mathbf{Q w} \mathbf{w} \\
= & \mathbf{J}(\mathbf{w}) \mathbf{f}(\mathbf{w})+\mathbf{J}(\mathbf{w}) \mathbf{J}^{\mathrm{H}}(\mathbf{w}) \hat{\mathbf{d}}+\lambda \widehat{\mathbf{a}}_{\mathrm{v}}+\gamma \mathbf{Q w} .
\end{aligned}
$$

We can obtain the offset vector $\overline{\mathbf{d}}$ :

$$
\hat{\mathbf{d}}=-\left[\mathbf{J}(\mathbf{w}) \mathbf{J}^{\mathrm{H}}(\mathbf{w})\right]^{-1}\left[\mathbf{J}(\mathbf{w}) \mathbf{f}(\mathbf{w})+\lambda \widehat{\mathbf{a}}_{\mathbf{v}}+\gamma \mathbf{Q w}\right] .
$$

Then, the updating weight vector for robust constrained LSCMA becomes

$$
\begin{aligned}
\mathbf{w}(k+1) & =\mathbf{w}(k)-\mathbf{G}(k)\left[\mathbf{X}_{K} \mathbf{e}^{\mathrm{H}}+\widehat{\mathbf{a}}_{\mathbf{v}} \lambda+\gamma \mathbf{Q} \mathbf{w}(k)\right] \\
& =(\mathbf{I}-\gamma \mathbf{G}(k) \mathbf{Q}) \mathbf{w}(k)-\mathbf{G}(k) \mathbf{X}_{K} \mathbf{e}^{\mathrm{H}}-\lambda \mathbf{G}(k) \widehat{\mathbf{a}}_{\mathbf{v}} .
\end{aligned}
$$

Substituting (31) into the linear constraint of (27), we can get

$$
\begin{aligned}
\lambda=\alpha & \left(\mathbf{p}^{\mathrm{T}} \mathbf{A}^{\mathrm{H}} \mathbf{w}(k)-\mathbf{p}^{\mathrm{T}} \mathbf{A}^{\mathrm{H}} \mathbf{G}(k) \mathbf{X}_{K} \mathbf{e}^{\mathrm{H}}\right. \\
- & \left.\gamma \mathbf{p}^{\mathrm{T}} \mathbf{A}^{\mathrm{H}} \mathbf{G}(k) \mathbf{Q w}(k)-1\right),
\end{aligned}
$$

where $\alpha=\left(\mathbf{p}^{\mathrm{T}} \mathbf{A}^{\mathrm{H}} \mathbf{G}(k) \mathbf{A p}\right)^{-1}$.

By inserting the multiplier $\lambda$ into (31), the weight vector can be rewritten as

$$
\begin{aligned}
\mathbf{w}(k+1) & \\
= & \mathbf{w}(k)-\mathbf{G}(k) \mathbf{X}_{K} \mathbf{e}^{\mathrm{H}}-\alpha \mathbf{G}(k) \mathbf{p}^{\mathrm{T}} \mathbf{A}^{\mathrm{H}} \mathbf{w}(k) \mathbf{A} \mathbf{p} \\
& +\alpha \mathbf{G}(k) \mathbf{p}^{\mathrm{T}} \mathbf{A}^{\mathrm{H}} \mathbf{G}(k) \mathbf{X}_{K} \mathbf{e}^{\mathrm{H}} \mathbf{A} \mathbf{p}+\alpha \mathbf{G}(k) \mathbf{A} \mathbf{p} \\
& -\gamma\left[\mathbf{G}(k) \mathbf{Q} \mathbf{w}(k)-\alpha \mathbf{G}(k) \mathbf{p}^{\mathrm{T}} \mathbf{A}^{\mathrm{H}} \mathbf{G}(k) \mathbf{Q w}(k) \mathbf{A} \mathbf{p}\right] .
\end{aligned}
$$

Inserting (33) into the quadratic constraint of (27), we can obtain

$$
\gamma=\frac{\operatorname{Re}\left[\mathbf{P}^{\mathrm{H}}(k) \mathbf{Q V}(k)\right]-\operatorname{Re}[\chi(k)]}{\mathbf{V}^{\mathrm{H}}(k) \mathbf{Q V}(k)},
$$

where

$$
\begin{aligned}
& \mathbf{P}(k)= \mathbf{w}(k)-\mathbf{G}(k) \mathbf{X}_{K} \mathbf{e}^{\mathrm{H}}-\alpha \mathbf{G}(k) \mathbf{p}^{\mathrm{T}} \mathbf{A}^{\mathrm{H}} \mathbf{w}(k) \mathbf{A} \mathbf{p} \\
&+\alpha \mathbf{G}(k) \mathbf{p}^{\mathrm{T}} \mathbf{A}^{\mathrm{H}} \mathbf{G}(k) \mathbf{X}_{K} \mathbf{e}^{\mathrm{H}} \mathbf{A} \mathbf{p}+\alpha \mathbf{G}(k) \mathbf{A} \mathbf{p}, \\
& \mathbf{V}(k)=\mathbf{G}(k) \mathbf{Q w}(k)-\alpha \mathbf{G}(k) \mathbf{p}^{\mathrm{T}} \mathbf{A}^{\mathrm{H}} \mathbf{G}(k) \mathbf{Q w}(k) \mathbf{A} \mathbf{p}, \\
& \chi^{*}(k) \chi(k)=\left(\operatorname{Re}\left[\mathbf{P}^{\mathrm{H}}(k) \mathbf{Q V}(k)\right]\right)^{2} \\
&-\left[\mathbf{V}^{\mathrm{H}}(k) \mathbf{Q V}(k)\left(\mathbf{P}^{\mathrm{H}}(k) \mathbf{Q P}(k)-\xi\right)\right] .
\end{aligned}
$$




\section{Performance Analysis}

4.1. The Implementation Complexity Cost. The complexity cost of the conventional LSCMA and the proposed robust LSCMA can be shown in Tables 1 and 2 .

4.2. Convergence Performance. The proposed robust constrained LSCMA is globally stable and convergent via Agee's inequalities. The first input stream is successfully extracted by establishing the following inequalities, given that $i>0$ [21]:

$$
\begin{gathered}
0 \leq\left\|\mathbf{y}_{1}(i)\right\|_{2}^{2} \leq\left\|\mathbf{y}_{1}(i+1)\right\|_{2}^{2} \leq K, \\
0 \leq\left\|\mathbf{y}_{1}(i+1)-\mathbf{y}_{1}(i)\right\|_{2}^{2} \leq g\left(\mathbf{w}_{1}(i)\right) \leq\left\|\mathbf{y}_{1}(i+1)\right\|_{2}^{2} \leq K, \\
0 \leq g\left(\mathbf{w}_{1}(i+1)\right) \leq g\left(\mathbf{w}_{1}(i)\right)-\left\|\mathbf{y}_{1}(i+1)-\mathbf{y}_{1}(i)\right\|_{2}^{2} \leq K,
\end{gathered}
$$

where $\mathbf{y}_{1}(i)=\mathbf{w}_{1}^{\mathrm{H}}(i) \mathbf{X}_{1}(i)$ and $\mathbf{X}_{1}(i)=\mathbf{X}_{K}$.

To extract the second input stream, we begin with the convergence of $\mathbf{X}_{2}(i)$ and then we have $\mathbf{y}_{2}(i)=\mathbf{w}_{2}^{\mathrm{H}}(i) \mathbf{X}_{2}(i)$, which is convergent via

$$
0 \leq\left\|\mathbf{y}_{2}(i)\right\|_{2}^{2} \leq\left\|\mathbf{y}_{2}(i+1)\right\|_{2}^{2} \leq K
$$

Then, each output stream is convergent via

$$
0 \leq\left\|\mathbf{y}_{m}(i)\right\|_{2}^{2} \leq\left\|\mathbf{y}_{m}(i+1)\right\|_{2}^{2} \leq K \text {. }
$$

For $i>0$, the overall performance may either improve or be maintained; thus the convergence performance of the proposed LSCMA is established.

4.3. Output SINR Performance. The output signal of the proposed beamformer can be expressed as

$$
\begin{aligned}
y(k) & =\mathbf{w}^{\mathrm{H}}(k) \mathbf{x}(k) \\
& =e^{j \varphi(k)}+\omega m(k) e^{j \phi(k)},
\end{aligned}
$$

where $\varphi(k)$ is the phase of the desired signal $s_{0}(k), \omega$ controls the SINR, and $m(k)$ and $\phi(k)$ are the magnitude and phase of the interference term, respectively. The normalized output is

$$
l(k)=\frac{y(k)}{|y(k)|}=\tau s_{0}(k)+\rho z(k)+v(k),
$$

where the parameters $\tau, \rho$ determine the power of the desired signal and the interference and $v(k)$ contains the intermodulation terms. Here we are interested only in the power of the desired signal and the interference in the normalized output, and the intermodulation terms are ignored.

Assuming that the initial beamformer SINR is known, the SINR of the normalized output signal $l(k)$ can be calculated [22]:

$$
\operatorname{SINR}=\frac{\tau^{2}}{\rho^{2}}=\frac{\left|E\left[s_{0}(k) l^{*}(k)\right]\right|^{2}}{\left|E\left[z(k) l^{*}(k)\right]\right|^{2}} .
$$

TABLE 1: The complexity cost of the conventional LSCMA.

\begin{tabular}{lc}
\hline & The complexity cost \\
\hline$\widehat{\mathbf{R}}_{K}$ & $O\left(M^{2} \times K\right)$ \\
$\widehat{\mathbf{R}}_{K}^{-1}$ & $O\left(M^{3}\right)$ \\
$\delta$ & $O\left(2 M^{2}+M \times K+2 M+K+1\right)$ \\
$\widehat{\mathbf{R}}_{K}^{-1} \mathbf{X}_{K} \mathbf{e}^{\mathrm{H}}$ & $O\left(M^{2} \times K+M \times K\right)$ \\
$\widehat{\mathbf{R}}_{K}^{-1} \mathbf{a}\left(\theta_{0}\right) \delta$ & $O\left(M^{2}+M\right)$ \\
\hline Total & $O\left(M^{3}+(2 K+3) \times M^{2}+(2 K+3) \times M+K+1\right)$ \\
complexity cost &
\end{tabular}

TABLE 2: The complexity cost of the proposed LSCMA.

\begin{tabular}{lc}
\hline & The complexity cost \\
\hline $\mathbf{Q}(k)$ & $O\left(4 M^{2}\right)$ \\
$\mathbf{G}(k)$ & $O\left(3 M^{2}+2 M\right)$ \\
$\mathbf{A p}$ & $O(M \times L)$ \\
$\mathbf{d}$ & $O\left(2 M^{2}+(K+L+2) \times M\right)$ \\
$\lambda$ & $O\left(M^{2}+4 M+2\right)$ \\
$\mathbf{P}(k)$ & $O\left(4 M^{2}+(K+1) \times M+K\right)$ \\
$\mathbf{V}(k)$ & $O\left(3 M^{2}+M\right)$ \\
$\chi(k)$ & $O\left(3 M^{2}+3 M+1\right)$ \\
\hline Total complexity cost & $O\left(20 M^{2}+(2 K+2 L+13) \times M+K+3\right)$ \\
\hline
\end{tabular}

We assume $\omega \ll 1$, so the cross-correlation of $s_{0}(k)$ and $l(k)$ is

$$
\begin{aligned}
\tau=E[ & \left(1+\omega m(k) e^{j(\varphi(k)-\phi(k))}\right) \\
& \times(1-\omega m(k) \cos (\varphi(k)-\phi(k)))] \simeq 1 .
\end{aligned}
$$

Similarly the cross-correlation of $z(k)$ and $l(k)$ is

$$
\begin{aligned}
\rho=E[ & {\left[m(k) e^{j(\phi(k)-\varphi(k))}+\omega m^{2}(k)\right) } \\
& \times(1-\omega m(k) \cos (\varphi(k)-\phi(k)))] \\
= & \frac{\omega}{2} .
\end{aligned}
$$

Inserting (43) and (44) to (42), we can obtain the output SINR as $4 / \omega^{2}$, so the normalized output of the proposed algorithm increases the array output SINR.

\section{Simulation Results}

In this section, Matlab software is used to evaluate the performance of the proposed algorithm. The sampling frequency is $F=3 f_{s}$ in the narrowband signal processing, where $f_{s}$ is the maximal signal frequency. The sine wave signal source has been used for simulations. The block diagram to clarify the simulation scenario is shown in Figure 1. A uniform linear array of $M=10$ sensors spaced half-wavelength apart is considered. All results are obtained by averaging 100 independent simulation runs. In all experiments, the nondirectional noise is assumed to be a spatially white Gaussian noise with unit covariance. It is assumed that there 


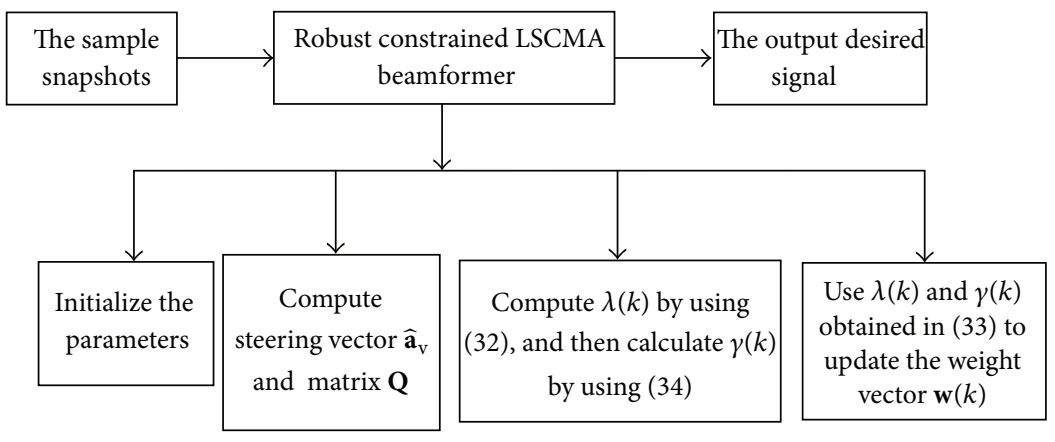

FIgURE 1: The simulation scenario diagram.

is one desired source at $3^{\circ}$ and two interfering sources at $-50^{\circ}$ and $50^{\circ}$. The a priori uncertainty in the DOA is over the region $u=\sin (\theta) \in[-0.2,0.2]$. The set $\Theta$ is composed of $L=20$ evenly spaced points on the interval $[-0.2,0.2]$. First, we show the performance of array beampattern. Next, we investigate the performance of SINR improvement brought by the proposed method. The constrained parameter $\xi^{2}=$ 0.03 is chosen for robust constrained LSCMA.

Example 1 (array beampatterns of two algorithms). The SNR is equal to $10 \mathrm{~dB}$. The aforementioned algorithms are simulated by using a mismatched steering vector of the desired signal, where the practical angle of incidence equals $6^{\circ}$. This corresponds to a $\Delta=3^{\circ}$ mismatch in the signal look direction. Figure 2 shows the array beampatterns of the above-mentioned algorithms against snapshots for the no mismatch case. The vertical line in the figure represents direction of arrival of the desired signal. It is clear that in the two algorithms deep nulls are formed at the directions of interferences and the array has magnitude response. The mismatch case is illustrated in Figure 3, where the vertical line represents the direction of the actual signal. We find out that at a small mismatch the linearly constrained LSCMA treats the desired signal as a main beam interferer and is trying to suppress it, which leads to performance degradation. However, the proposed algorithm is better at providing robustness and having resolution compared with the linearly constrained LSCMA.

Example 2 (output SINR versus snapshots). In the second experiment, the SINR performance of the aforesaid algorithms for the fixed SNR $=0 \mathrm{~dB}$ against the array imperfections is illustrated in Figures 4 and 5. In this example, the constrained LSCMA is very sensitive even to slight mismatches, which can cause signal cancellation problem. The result in Figure 5 shows that the proposed method offers about $5 \mathrm{~dB}$ improvement over the linearly constrained LSCMA and makes output SINR close to the optimal one due to the efficient handling of the average steering vector and worst-case performance optimization. It is clear that the proposed algorithm has superior performance on SINR improvement for no array imperfections.

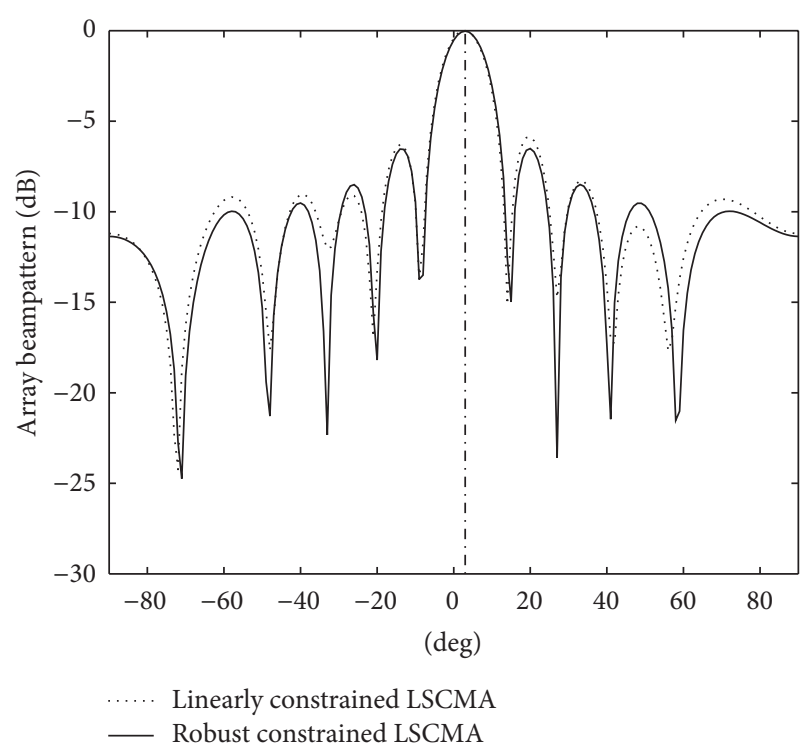

FIgURE 2: Array beampattern (in no mismatch case).

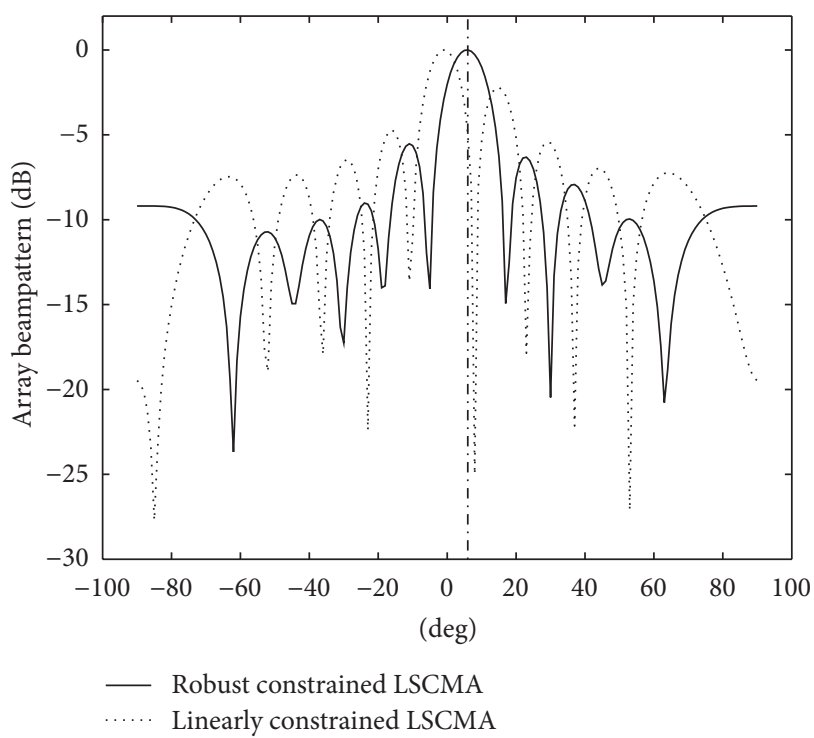

FIGURE 3: Array beampattern (in the mismatch case). 


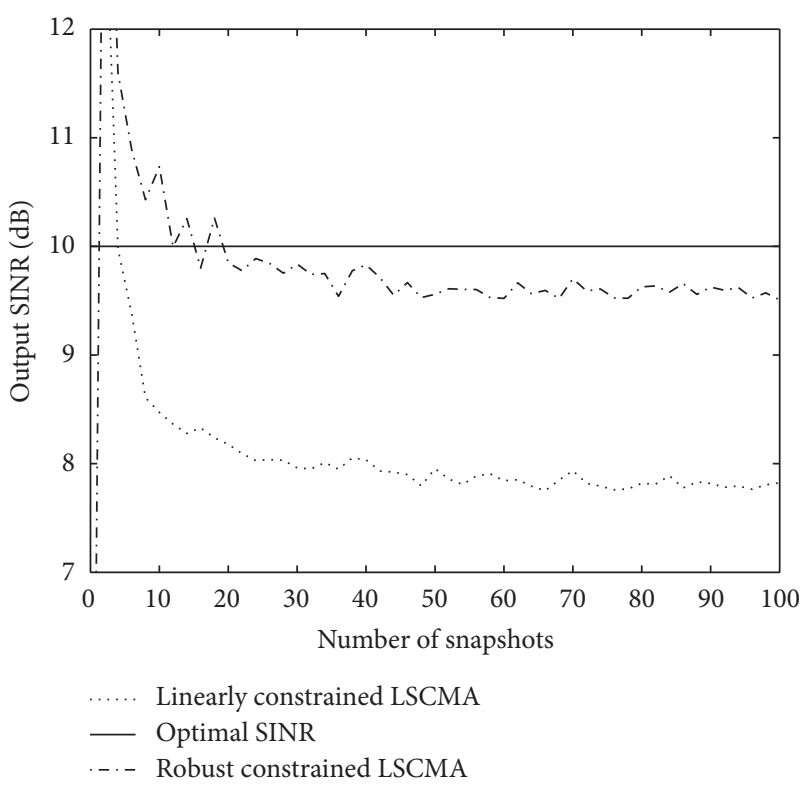

FIgURE 4: Output SINR versus $N$ (in no mismatch case).

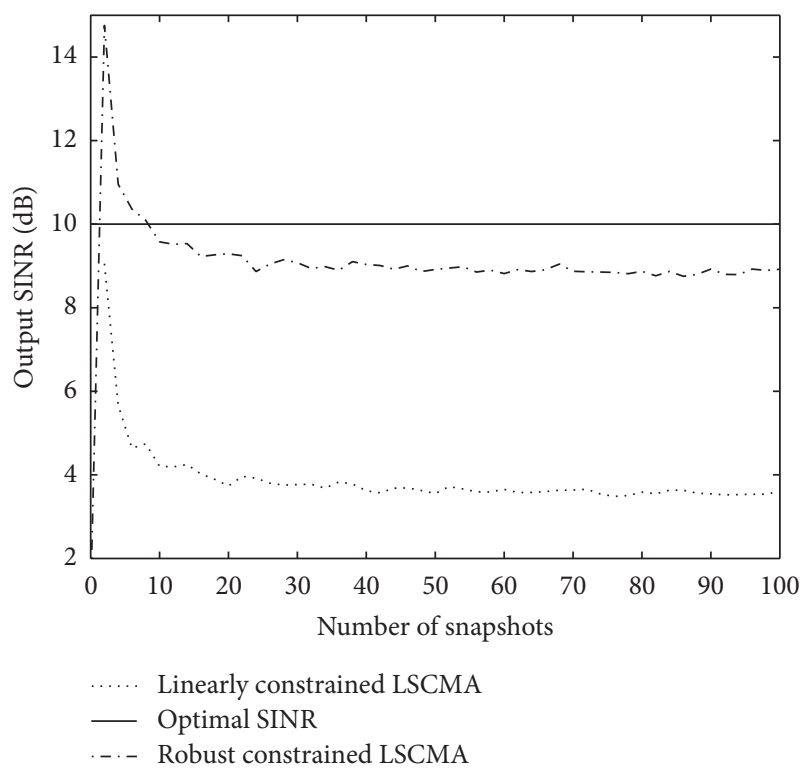

FIGURE 5: Output SINR versus $N$ (in the mismatch case).

Example 3 (output SINR versus input SNR). In this experiment, we evaluate the SINR performance versus input SNR with DOA error for the fixed sample data size $N=100$. The simulation results in Figures 6 and 7 indicate that the proposed method has slight performance degradation with the increasing of input SNR, and it is not sensitive to the power of the target signal. In this example, the performance of the linearly constrained LSCMA degrades when the signal power increases. Robust constrained LSCMA is known theoretically not to reach the optimal performance, but it is seen that for positive SNR the performance is almost identical to the optimal SINR. As expected, the sensitivity

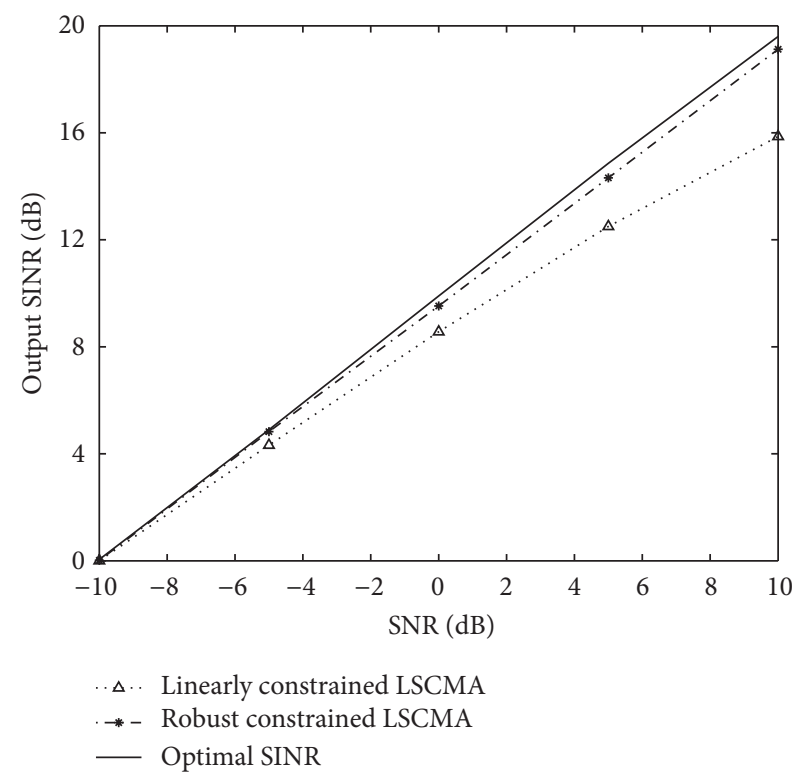

Figure 6: Output SINR versus SNR (in no mismatch case).

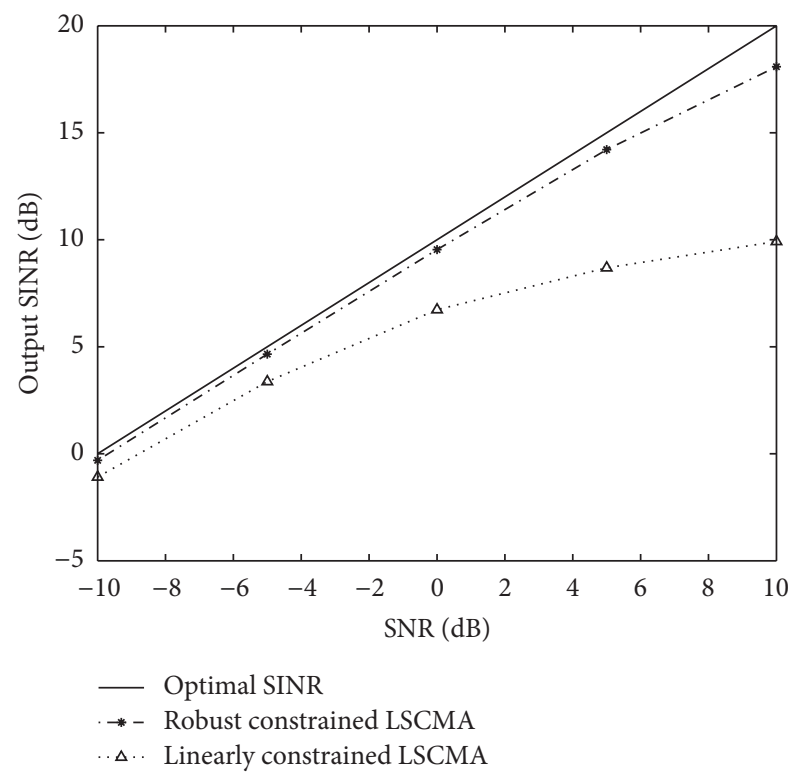

FIgURE 7: Output SINR versus SNR (in the mismatch case).

to signal steering vector mismatches can be significantly lowered by the proposed algorithm. As a result, the proposed algorithm can provide sufficient robustness to pointing errors in perturbation situations.

\section{Conclusions}

In this paper, a novel robust LSCMA algorithm based on double constraints is proposed via the Bayesian approach and worst-case performance optimization. To improve robustness, the weight vector is optimized to involve minimization of the objective function with penalty for the worst-case 
signal steering vector by the partial Taylor-series expansion and Lagrange multiplier method, in which the parameters can be precisely derived at each iterative step. Moreover, the online implementation of the proposed algorithm eliminates the covariance matrix inversion estimation, which has a low computational load. The proposed robust constrained LSCMA has a faster convergence rate, provides better robustness against the signal steering vector mismatches, and yields improved array output performance compared with the linearly constrained LSCMA. The theoretical analysis and simulation experiments have been carried out to illustrate the significant performance improvement of the proposed method for the signal steering vector mismatches.

\section{Acknowledgments}

The authors would like to thank the anonymous reviewers for their insightful comments that helped improve the quality of this paper. This work was supported by the Program for New Century Excellent Talents in University no. NCET-12-0103, the Natural Science Foundation of Liaoning province under Grant no. 201102057, and the Natural Science Foundation of Hebei province under Grant no. F2012501044.

\section{References}

[1] X. Huang, H.-C. Wu, and J. C. Principe, "Robust blind beamforming algorithm using joint multiple matrix diagonalization," IEEE Sensors Journal, vol. 7, no. 1, pp. 130-135, 2007.

[2] S. A. Farès, T. A. Denidni, S. Affès, and C. Despins, "Fractionaldelay sequential blind beamforming for wireless multipath communications in confined areas," IEEE Transactions on Wireless Communications, vol. 7, no. 2, pp. 629-638, 2008.

[3] J. Yang, H. Xi, F. Yang, and Y. Zhao, "Fast adaptive blind beamforming algorithm for antenna array in CDMA systems," IEEE Transactions on Vehicular Technology, vol. 55, no. 2, pp. 549-558, 2006.

[4] N. Xie, Y. Zhou, M. Xia, and W. Tang, "Fast blind adaptive beamforming algorithm with interference suppression," IEEE Transactions on Vehicular Technology, vol. 57, no. 3, pp. 19851988, 2008.

[5] X. Song, J. Wang, and B. Wang, "Robust adaptive beamforming under quadratic constraint with recursive method implementation," Wireless Personal Communications, vol. 53, no. 4, pp. 555$568,2010$.

[6] B. D. van Veen, "Minimum variance beamforming with soft response constraints," IEEE Transactions on Signal Processing, vol. 39, no. 9, pp. 1964-1972, 1991.

[7] K. L. Bell, Y. Ephraim, and H. L. van Trees, "A Bayesian approach to robust adaptive beamforming," IEEE Transactions on Signal Processing, vol. 48, no. 2, pp. 386-398, 2000.

[8] D. D. Feldman and L. J. Griffiths, "Projection approach for robust adaptive beamforming," IEEE Transactions on Signal Processing, vol. 42, no. 4, pp. 867-876, 1994.

[9] L. Chang and C.-C. Yeh, "Performance of DMI and eigenspacebased beamformers," IEEE Transactions on Antennas and Propagation, vol. 40, no. 11, pp. 1336-1348, 1992.

[10] J. Yang, X. Ma, C. Hou, and Y. Liu, "Automatic generalized loading for robust adaptive beamforming," IEEE Signal Processing Letters, vol. 16, no. 3, pp. 219-222, 2009.
[11] J. Li, P. Stoica, and Z. Wang, "Doubly constrained robust Capon beamformer," IEEE Transactions on Signal Processing, vol. 52, no. 9, pp. 2407-2423, 2004.

[12] S. A. Vorobyov, A. B. Gershman, and Z.-Q. Luo, "Robust adaptive beamforming using worst-case performance optimization: a solution to the signal mismatch problem," IEEE Transactions on Signal Processing, vol. 51, no. 2, pp. 313-324, 2003.

[13] A. Elnashar, S. M. Elnoubi, and H. A. El-Mikati, "Further study on robust adaptive beamforming with optimum diagonal loading," IEEE Transactions on Antennas and Propagation, vol. 54, no. 12, pp. 3647-3658, 2006.

[14] A. Gershman, N. Sidiropoulos, S. Shahbazpanahi, M. Bengtsson, and B. Ottersten, "Convex optimization-based beamforming," IEEE Signal Processing Magazine, vol. 27, no. 3, pp. 62-75, 2010.

[15] A. Elnashar, "Efficient implementation of robust adaptive beamforming based on worst-case performance optimisation," IET Signal Processing, vol. 2, no. 4, pp. 381-393, 2008.

[16] S. Shahbazpanahi, A. B. Gershman, Z.-Q. Lou, and K. M. Wong, "Robust adaptive beamforming for general-rank signal models," IEEE Transactions on Signal Processing, vol. 51, no. 9, pp. 22572269, 2003.

[17] E. A. Gharavol, Y.-C. Liang, and K. Mouthaan, "Robust downlink beamforming in multiuser MISO cognitive radio networks with imperfect channel-state information," IEEE Transactions on Vehicular Technology, vol. 59, no. 6, pp. 2852-2860, 2010.

[18] A. Mukherjee and A. L. Swindlehurst, "Robust beamforming for security in MIMO wiretap channels with imperfect CSI," IEEE Transactions on Signal Processing, vol. 59, no. 1, pp. 351-361, 2011.

[19] M. Rübsamen and A. B. Gershman, "Robust adaptive beamforming using multidimensional covariance fitting," IEEE Transactions on Signal Processing, vol. 60, no. 2, pp. 740-753, 2012.

[20] B. G. Agee, "The least-squares CMA: a new technique for rapid correction of constant modulus signal," in Proceedings of Acoustics, Speech and Signal Processing, pp. 953-956, Tokyo, Japan, April 1986.

[21] P. Sansrimahachai, D. B. Ward, and A. G. Constantinides, "Blind source separation of instantaneous MIMO systems based on the least-squares constant modulus algorithm," IEE Vision, Image and Signal Processing, vol. 152, pp. 616-622, 2005.

[22] T. E. Biedka, W. H. Tranter, and J. H. Reed, "Convergence analysis of the least squares constant modulus algorithm in interference cancellation applications," IEEE Transactions on Communications, vol. 48, no. 3, pp. 491-501, 2000. 


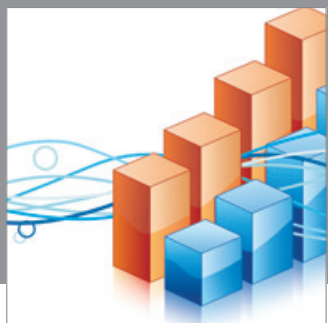

Advances in

Operations Research

mansans

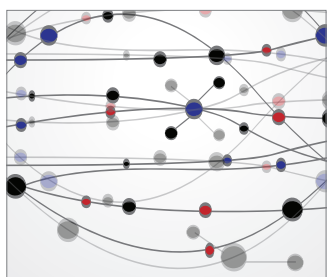

The Scientific World Journal
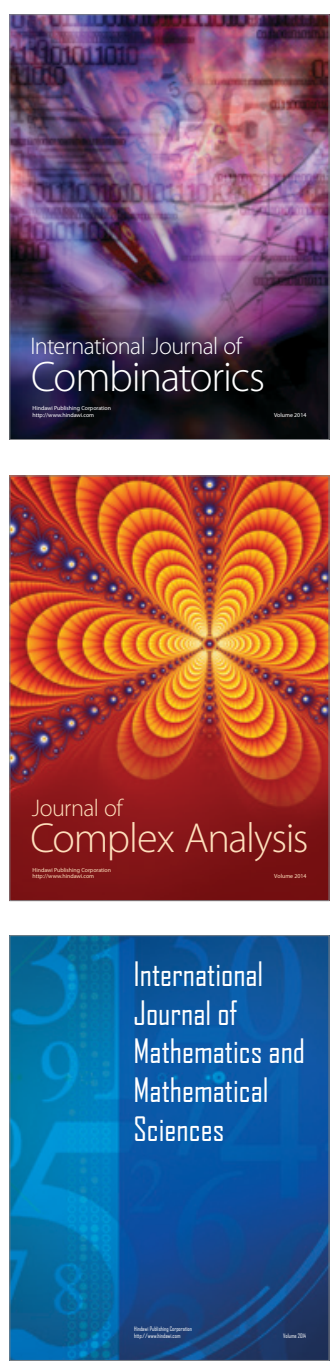
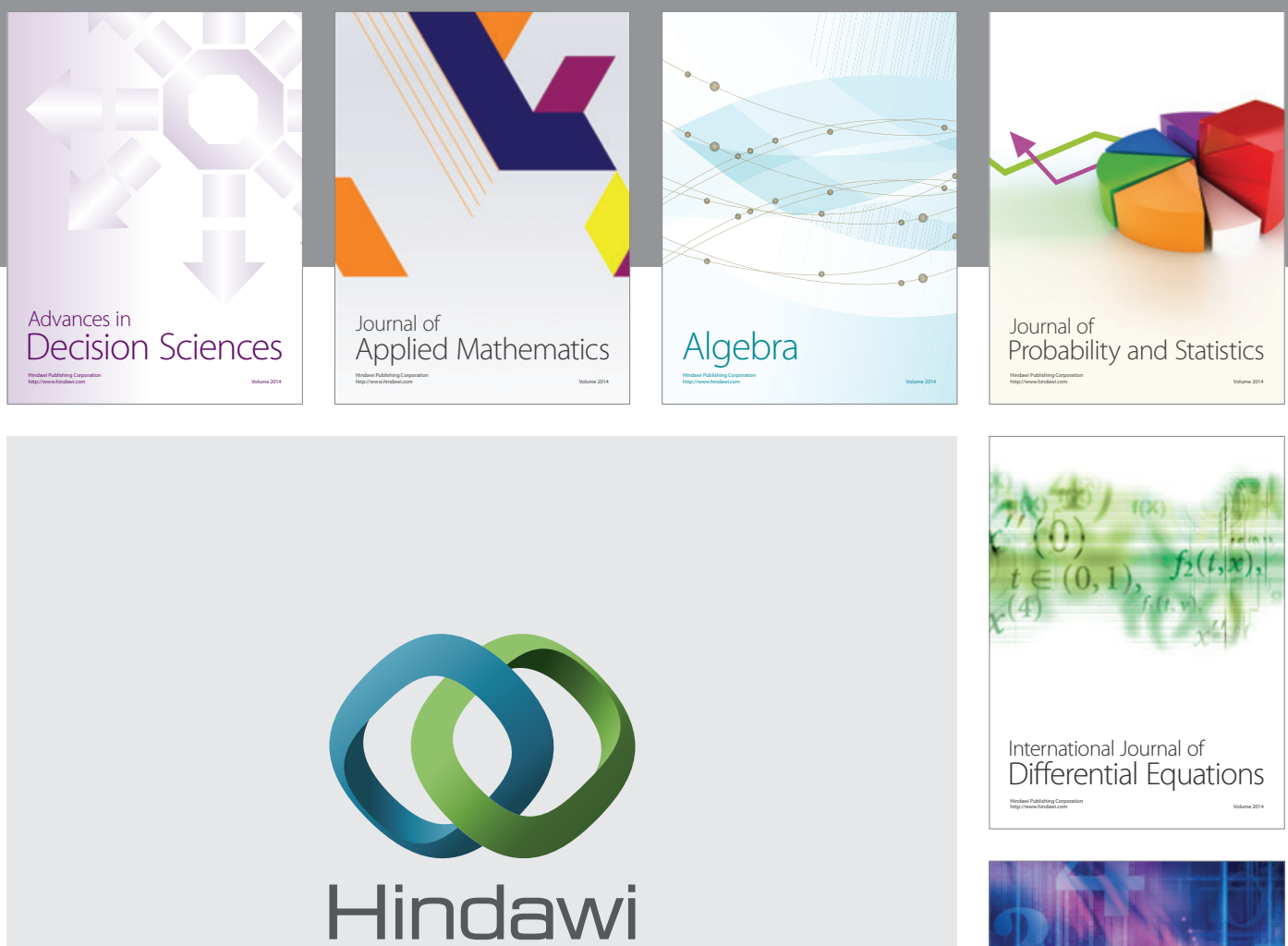

Submit your manuscripts at http://www.hindawi.com
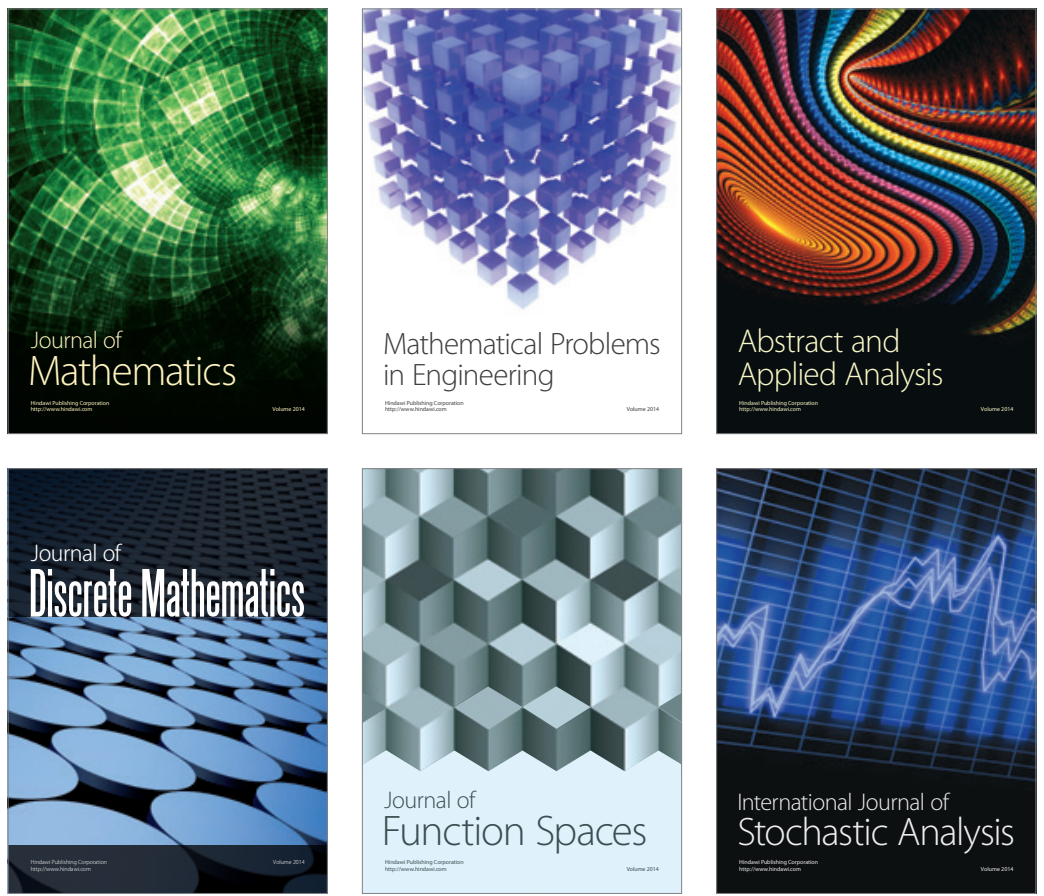

Journal of

Function Spaces

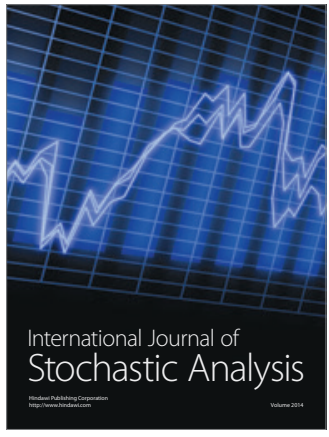

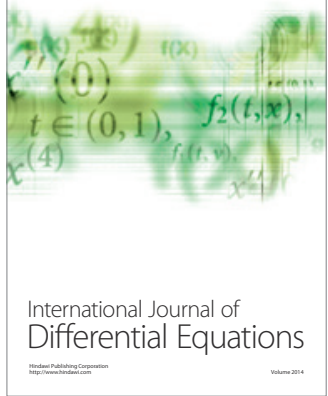
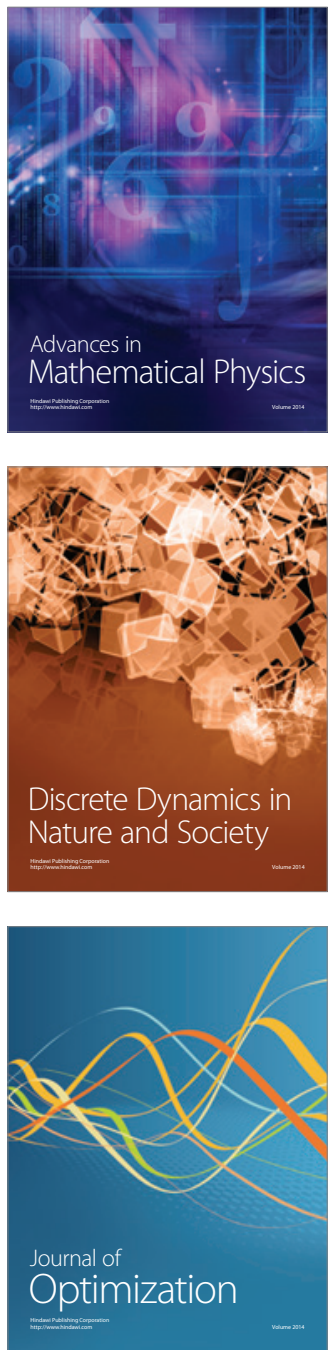